\title{
Electric Railway Loufan Traction Station Of Shanxi Power Grid Harmonic
}

\section{Impact Analysis}

\author{
Mingde Cao ${ }^{1}$, Jinhao Wang ${ }^{1}$, Da Lei ${ }^{1}$, Chaoying Yang ${ }^{1}$, Lei Feng ${ }^{1}$, \\ Lei $\mathrm{Yao}^{2}$, Weiqing Sun² \\ 1Electric Power Research Institute, State Grid Shanxi electric power company, \\ Taiyuan, Shanxi Province, 030001, China \\ ${ }^{2}$ School Optical-Electrical and Computer Engineering, University of Shanghai for \\ Science and Technology, Shanghai, 200093, China
}

Keywords: Electric locomotive; Harmonic analysis; Power quality; Traction station

Abstract: With the rapid development of Shanxi electric railway, the harmonic problem is also more serious. In order to analyze the harmonic problems caused by the new Loufan traction station. In this paper, the main electric locomotive to simulate is SS4G in Loufan traction station. The simulation and test results show that the harmonic distribution of electric locomotive works in the first stage with the larger change of the switching angle. When the switching angle is about 40 degrees, the harmonic content is the smallest., because electric locomotive work during the second and third sections all-pass state as part of the bridge circuit, lead to small harmonic content changeand keep in a larger value. Therefore, when the electric locomotive works in the three and second sections, But by changing the all-pass bridge circuit opening angle to reduce the harmonic.

\section{Introduction}

Railway is the main artery of the national economy, which bears more than $50 \%$ of the country's passenger and cargo traffic ${ }^{[1]}$. Electrified railway using electric traction, with higher energy efficiency, greater power and traction, it can drive heavier loads, moreover to improve the lot of speed than diesel locomotive, so as to enhance the efficiency of the entire community of travelers ${ }^{[2]}$.

However, electric locomotive possesses nonlinear, asymmetric and impact resistance characteristics, bring harmonic, negative sequence, voltage offset and flicker to the grid. This caused great impact on the deterioration of power quality ${ }^{[3,4]}$.

There are a lot of coal resources in Shanxi province, it requires a lot of heavy-duty electric railway transport, therefore, Shanxi Electric Railway is one of the main sources of pollution in Shanxi Power Grid, Xinzhou, Datong and other regions have more than the power grid events caused by electric iron operation., this threatens the stable and secure operation of the grid and causes serious damage to the electrical equipment ${ }^{[5,6]}$.

Tai Hing is a railway in Shanxi's coal transport, both freight supplemented by regional trunk railway, Starting point is Fenhe of Taiyuan North Marshalling Station, finishing point is baiwen station of South central Shanxi Railway Corridor, and across the board (freight) traffic in December 30, 2014. Loufan traction station, which is Tai Hing Railway traction station set for Loufan County. State Grid Taiyuan power supply company provides two 110-kilovolt line for Loufan traction station, length of $46.34 \mathrm{~km}$. This solves the problem of electricity demand of Tai Hing Railway. 
Freight traction trains mainly to SS4G in Tai Hing Railway. In order to analyze the impact on the power grid that Loufan traction station brings, especially on harmonics. This paper to make a woke in SS4G harmonic simulation analysis, it can provide the basis for the grid in governance harmonics.

\section{SS4G electric locomotive main circuit and working principle}

Electric railway locomotive model is SS4G in Loufan traction station. SS4G electric locomotive circuit (see figure one) is based on SS4, SS5 and SS6 locomotive main circuit, and digestion and absorption of $8 \mathrm{~K}$ and $6 \mathrm{~K}$ locomotive of some advanced technology and design.

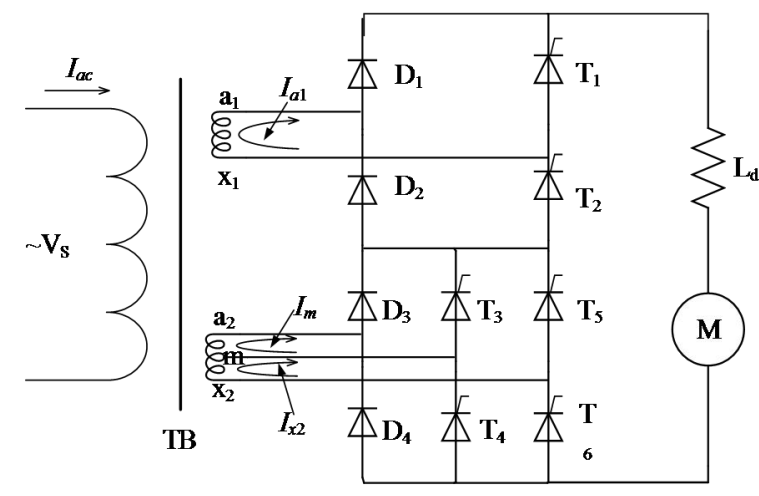

\section{Figure 1 SS4G electric locomotive main circuit diagram}

Schematic diagram shown in Figure 1, AC Vc obtained energy by pantograph connection from the traction station, through transformer $\mathrm{TB}$, get low-side voltage $U_{\mathrm{a} 1 \times 1}=700 \mathrm{~V}, U_{\mathrm{a} 2 \mathrm{~m}=} U_{\mathrm{a} 2 \times 2}=350 \mathrm{~V}$. Through the bridge circuit to obtain a DC voltage and supply traction DC motor M, Ld is DC-side smoothing inductor. The working process of the bridge circuit to the following three sections.

1. control T1, T2 trigger conduction, T3 T5 are in ending state control, not consider AC inductance and the angular commutation influence, the output voltage of the DC side by side equivalent a1x1 D1, D2, T1, T2 half-controlled rectifier to get. Its DC voltage and current waveforms $27.5 \mathrm{kV}$ side shown in FIG. 2 (a).

2. T1, T2 has been in a conducting state control T3, T4 trigger conduction, T5, T6 is controlled in ending state, not consider AC inductance and the angular commutation influence, At this time of the output voltage of the DC side by side equivalent a1x1 D1, D2, T1, T2 consisting of uncontrolled rectifier superimposed a2m side by D3, D4, T3, T4 composed of half-controlled rectifier to get. Its DC voltage and current waveforms $27.5 \mathrm{kV}$ side shown in FIG. 2 (b).

3. T1, T2, T3, T4 has been in a conducting state control T5, T6 trigger conduction, not consider $\mathrm{AC}$ inductance and the angular commutation influence, the DC output voltage is divided into two cases: (1) When T5, T6 not trigger conduction, the DC output voltage is equivalent a1x1 side by D1, D2, T1, T2 consisting of uncontrolled rectifier superimposed a2m side by D3, D4, T3, T4 consisting of a rectifying circuit to get. (2) When T5, T6 trigger conduction, due to the T3, T4 is reverse biased, in an off state, the output voltage of the DC side by side equivalent a1x1 D1, D2, T1, T2 consisting of uncontrolled rectifier superimposed a2x2 side by D3, D4, T5, T6 composed of a rectifying circuit to get. Its DC voltage and current waveforms $27.5 \mathrm{kV}$ side shown in FIG. 2 (c). According to the figure shows, this process can be equivalent to two working uncontrolled rectifier is a2m side by D3, D4, T3, T4 composed of superimposed mx2 side by T3, T4, T5, T6 forming a semi-controlled rectifier, wherein T3, T4 equivalent has been in a conducting state. 


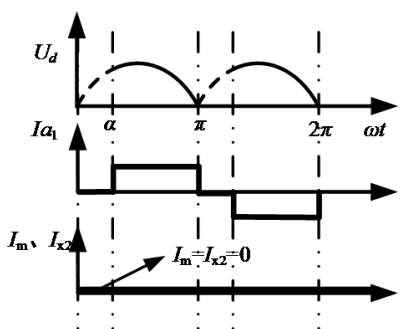

(a) The First stage

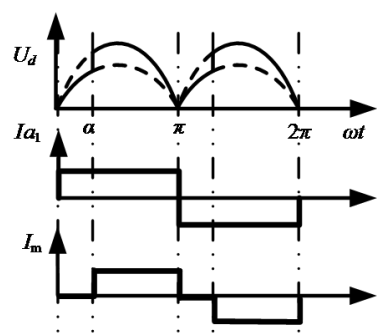

(b) The second stage

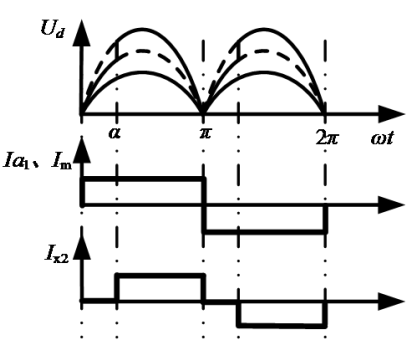

(c) The third stage

Figure2 Electric locomotive operating voltage and current

Figure 2 shows the voltage curve and the bridge in three segments corresponding to each of the voltage.

\section{Harmonic Analysis of SS4G electric locomotives}

As the figure 1 shows, high-side current by the superposition of $I_{a 1}, I_{m}$ and $I_{x 2}$ through the transformer, $I_{a 1}, I_{m}$ and $I_{x 2}$ current waveform shown in Figure 2, the figure shows that $I_{a 1}, I_{m}$ and $I_{x 2}$ are symmetrical waveform points, symmetrical points $(0,0)$ or $(\alpha / 2,0)$, Therefore, the amplitude of each current harmonics:

$$
I_{n}=\frac{1}{\pi} \int_{-\pi}^{\pi} i(\varpi t) \sin (n \varpi t) d(\varpi t)=\frac{2}{\pi} \int_{0}^{\pi} i(\varpi t) \sin (n \varpi t) d(\varpi t)
$$

For the second-stage $I_{a 1}$ and third stage $I_{a 1}, \mathrm{I}_{m}$ a square wave signal, the peak of each harmonic

$$
I_{n}=\frac{2}{\pi} \int_{0}^{\pi} I_{d} \sin (n \varpi t) d(\varpi t)=\left\{\begin{array}{l}
\frac{4 I_{d}}{n \pi}, n \text { odd } \\
0, n \text { even }
\end{array}\right.
$$

For the first stage $I_{a 1}$, and the second $\mathrm{I}_{m}$ square wave signal and the third paragraph $I_{x 2}$, waveform observation shows that its point of symmetry $(\alpha / 2,0)$, therefore the magnitude of its harmonics are as follows:

$$
I_{n}=\frac{2}{\pi} \int_{\frac{\alpha}{2}}^{\pi-\frac{\alpha}{2}} I_{d} \sin (n \varpi t) d(\varpi t)=\left\{\begin{array}{c}
\frac{4 I_{d d}}{n \pi} \cos \frac{n \alpha}{2}, n \text { odd } \\
0, n \text { even }
\end{array}\right.
$$

AC voltage side phase difference

$$
\varphi_{n}=n \frac{\alpha}{2}
$$

Wherein $I_{d}$ is a direct current.

Thus, without considering the influence of the leakage inductance and magnetizing circuit case, the high side current:

The first stage of the n-th harmonic current:

$$
I_{n}^{(1)}=\frac{I_{\text {an1 }}}{N}=\left\{\begin{array}{c}
\frac{4 I_{d}}{n \pi N} \cos \frac{n \alpha}{2}, n \text { odd } \\
0, n \text { even }
\end{array}\right.
$$

The second stage of the $\mathrm{n}$-th harmonic current:

$$
I_{n}^{(2)}=\sqrt{\left(\frac{I_{\text {al }}}{N}\right)^{2}+\left(\frac{I_{m}}{2 N}\right)^{2}+2 \frac{I_{\text {an }}}{N} \frac{I_{m}}{2 N} \cos \varphi_{n}}=\left\{\begin{array}{c}
\frac{4 I_{d d}}{n \pi N} \sqrt{1+\frac{5}{4} \cos ^{2} \frac{n \alpha}{2}}, n \text { odd } \\
0, n \text { even }
\end{array}\right.
$$

The third stage of the n-th harmonic current: 


$$
I_{n}^{(3)}=\sqrt{\left(\frac{I_{m 1}}{N}+\frac{I_{m}}{2 N}\right)^{2}+\left(\frac{I_{N 2}}{2 N}\right)^{2}+2\left(\frac{I_{m 1}}{N}+\frac{I_{m}}{2 N}\right) \frac{I_{N 2}}{2 N} \cos \varphi_{n}}=\left\{\begin{array}{c}
\frac{4 I_{d}}{n \pi N} \sqrt{\frac{9}{4}+\frac{7}{4} \cos ^{2} \frac{n \alpha}{2}}, n \text { odd } \\
0, n \text { even }
\end{array}\right.
$$

After calculation available, the harmonic content of each section of the work are shown in the figure. Wherein abscissa is the harmonic number, the value range of 3 to 50, the ordinate of each harmonic with respect to the fundamental amplitude.

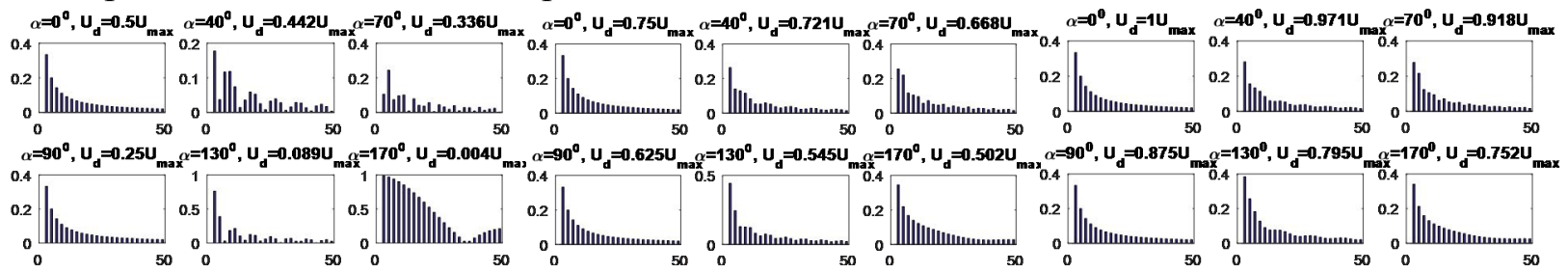

(a) The first stage

(b) The second stage

(c) The third stage

Figure 3: Electric locomotive in the harmonic content of each stage

Figure (a) shows that electric locomotive works in The first stage, harmonics greatly influenced by switching angles. With the increase of the angle switch, harmonic content decreased when $\alpha$ is about $40^{\circ}$, the harmonic content is minimal, Wherein the fifth harmonic content is less; With the switching angle $\alpha$ is further increased, the harmonic content begins to increase, the fifth harmonic content is less when $\alpha$ is about $70^{\circ}$ 。 When $\alpha=90^{\circ}$ and $\alpha=0^{\circ}$, the harmonic content are basically the same, when the switch $\alpha$ approaching $180^{\circ}$, the harmonic content were higher.

Figure (b) (c) shows that electric locomotive in the second stage and the third paragraph of each harmonic content, there is little affected by the switch of the angle $\alpha$, The main reason is D1, D2, T1, T2 composed bridge full-wave rectification state, The ratio of primary voltage and the voltage of the $\mathrm{AC}$ winding which connected to this bridge is $\mathrm{N}$, It accounted for the major part of the high-pressure side of the current, Therefore, the harmonic content is mainly controlled by this bridge.

\section{Harmonic analysis of data generated by loufan traction station on the grid}

To further study the impact on the grid harmonic electric locomotive, the research group at January 27, 2016 16:42:30 until February 1, 2016 12:16:30 loufan traction station on the grid side station detects, loufan traction station have been SS4G freight electric locomotive. Remove the three time points, and the harmonic content of the current corresponding to the simulation data shown in figure 4 , where the abscissa is 3 to 50 times each harmonic frequency, the vertical axis is relative to the fundamental harmonic current current oil content.

Figure 4(a) is the tharmonic content ratio when electric locomotive work in The first stage, Compare electric locomotive switching angle $\left(\alpha=66^{\circ}\right)$ can be observed in its harmonic ratio is closer, few three times harmonic, fifth harmonic and the rest were higher, less other high harmonic content .

Figure 4(b)and figure 4(c) are the electric locomotive harmonic ratio, when it works on the second stage and the third stage. Third harmonic larger, and with the increasing number of harmonics, the harmonic content is reduced. Compared with the calculated data, each harmonic content are small, This is mainly because the locomotive installed PFC in order to improve locomotive power factor and improve communications interference. 


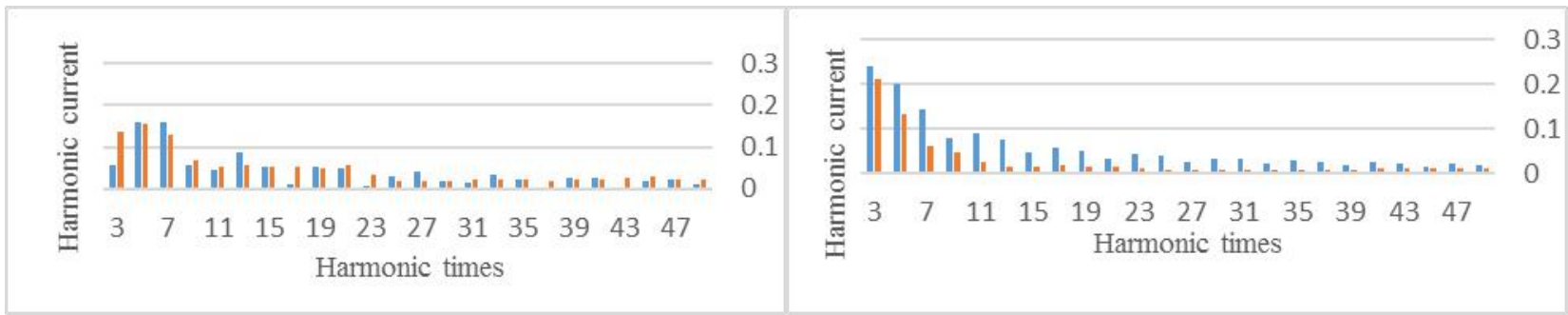

(a) The first stage

(b) The second stage

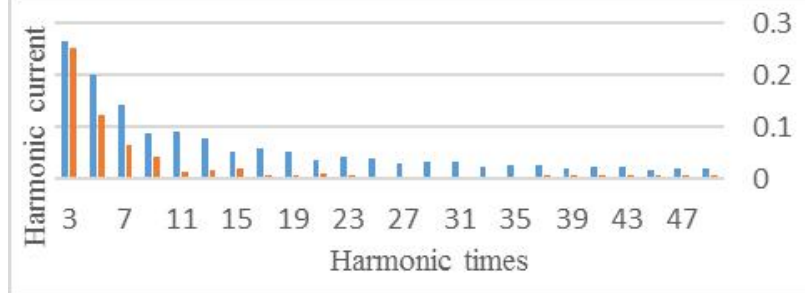

(c) The third stage

Figure4: Electric locomotive harmonics measured and simulated data

\section{Conclusion}

The electric locomotive mainly through Loufan traction station are SS4G. When electric locomotives working in the first stage and the switch angle $\alpha$ is about $40^{\circ}$, harmonics are minimum. When working in second and third stage, some bridge in all the conduction state, switching angles change has little effect on the harmonic, data maintained in a larger context. Therefore, when the electric locomotive in the second and third stages, changing the surge strategy to reduce the harmonic content of the system, so that in the whole state of the bridge there is a certain angle switch. Harmonics can be reduced only by a simple control strategy change.

\section{Acknowledgements}

This work is supported by National grid science and technology project

\section{References}

[1] Zhao Wenlei, Kong Li, Wang Juanjuan, Zhen Xia. Harmonic analysis based on MATLAB and wavelet transform for electrical locomotive[J], Electric Power Automation Equipment, 2012,32(1):103-106.

[2] Lin Gao, Yonghai Xu, Xiangnin Xiao, etc. Simulation model and harmonic analysis of SS6B electric locomotive based on PSCAD/EMTDC[C], 2008 IEEE Electrical Power and Energy Conference - Energy Innovation, 2008.

[3] Dong, Xiang, Li, Qun-Zhan, Huang, Jun, etc.Detecting methods of reactive power and harmonic current in electrified railway systems[J], Power System Protection and Control, 2009,37(6):32-35.

[4] Fan, Chun-Lei, Wu, Guang-Ning, Zhang, Xue-Yuan, etc. The measurement and simulation research of electrified railway harmonic[C], Proceedings of 2008 International Conference on Condition Monitoring and Diagnosis, CMD 2008, p 769-772.

[5] WANG Ye, YANG Xiao-guo. Brief Discussion on the Effect of Electrification Railway Load to Power Quality of Shanxi Power Grid [J]. China Electric Power(Technology Edition), 2013,(7):25-27.

[6] ZHU Yanfang,WU Xiaodong. Analysis of the status Quo of Electrified Railway and Its Construction Planning in Shanxi [J]. Shanxi Electric Power, 2011, (1):1-3. 